\title{
Modeling solar magnetoconvection and coronal structures
}

\author{
Neal E. Hurlburt and Marc L. Derosa \\ Lockheed Martin Solar and Astrophysics Laboratory \\ $\mathrm{O} / \mathrm{ADBS}, \mathrm{B} 252$ \\ 3251 Hanover St, Palo Alto, CA 94304
}

\begin{abstract}
We present results of an investigation into the coupling between solar-like magnetoconvection and coronal structures using self-consistent numerical simulations of compressible fluids. The model consists of a stratified MHD fluid spanning multiple scale heights, encompassing the transition of the plasma beta from high to low values. The lower portion of the domain, where the beta is high, is convectively unstable while the upper portion is stabilized by the presence of a strong magnetic field and energy losses. As a result, the dynamics associated with evolving magnetic features in the solar atmosphere can be modelled in a manner that is self-consistent with the convective motions that provide the driving. We present simulations of arcade-like reconnection in the presence of supergranular-scale flows.
\end{abstract}

The dynamic coupling between evolving magnetic structures visible in the solar corona and the vigorous fluid motions that inhabit the solar convection zone is just beginning to be understood. The turbulent convection below the photosphere continually redistributes the magnetic field, as magnetic field is stretched, twisted, and otherwise reorganized in response to fluid motions. Such dynamics influence the structure and heating of the solar corona by laterally advecting the photospheric footpoints of coronal magnetic loops, thereby causing changes in the coronal field topology that may ultimately trigger flares, coronal mass ejections, and other eruptive events.

In this poster, we present results of two-dimensional numerical simulations of fully compressible, magnetized fluids in spherical geometry. An initial large-scale bipolar magnetic field is imposed within localized spherical segments, simulating a fully emerged active region. Convection on the (approximate) scale of solar supergranulation is driven by imposing a temperature flux at the lower boundary of the domain. We investigate the resulting dynamics within our simulation domain.

Using the fully convective simulations as a jumping-off point (DeRosa and Hurlburt 2003), we have extended the upper radial boundary of the simulation to include a passively heated layer above the convection, thereby approximating the corona of the sun. Such simulations enable the magnetic loops in the corona to evolve in a self-consistent manner as their respective footpoints are advected by fluid motions within the convection zone.

We solve the fully compressible MHD equations for a non-rotating spherical segment. The equations are nondimensionalized using the depth of the convecting layer $\mathrm{d}=\mathrm{R} 2-\mathrm{R} 1$ and the state variables $\rho_{2}, T 2$, and $P_{2}$ at the upper boundary. Time is measured in units of the isothermal sound crossing time at the upper boundary, $d / v\left(R^{*} T_{2}\right)$, where $R^{*}$ is the gas constant. The radial boundaries of the convecting layer are located at $R_{1}=24$ and $R_{2}=25$, and may be extended to include a model corona with an upper radius $R_{3}=26$. The outer boundaries and impenetrable and stress-free. The latitudinal sidewalls at $T_{1}$ and $T_{2}$ are assumed impenetrable and stress-free, and the domain is periodic in longitude. A temperature gradient $\Theta=10$ is applied across the convection zone, while the upper 


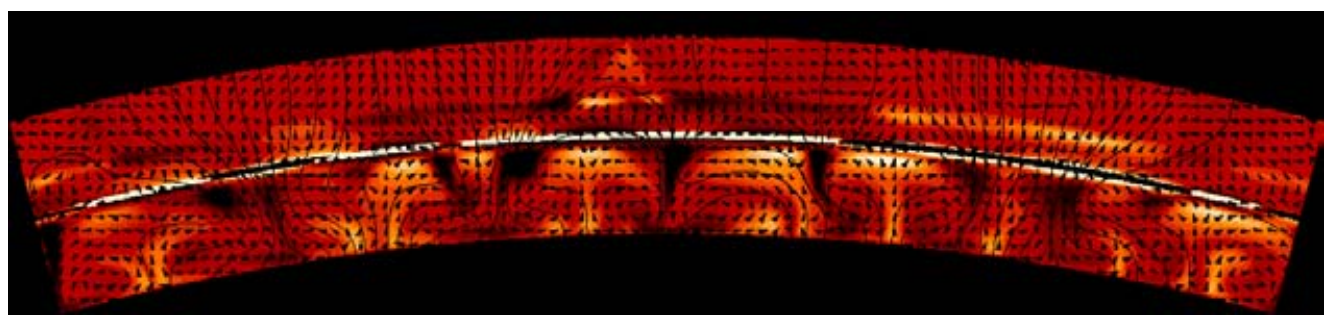

Figure 1. A 2D simulation encompassing idealized layers of convectively unstable layer, a cooled chromosphere and heated corona. Here the flow direction (not magnitude) is displayed as arrows, the magnetic field as lines, and the entropy as the background shading.

boundary is held at constant temperature. The stratification of the convecting layer is set by the polytropic index $m=1.45$, while in the atmosphere matching exponentials are strung together in a piecewise fashion. The gas has a $\gamma=5 / 3$. The magnetic field is assumed to be purely radial at both radial boundaries, while the latitudinal sidewalls are assumed to be perfectly conducting. Solving the fully compressible MHD equations within a curved spherical segment allows us to investigate the dynamics associated with the evolution of bipolar magnetic flux configurations. Our aim is to provide insight into the evolution of a magnetized fluid (such as solar active region), and to understand how such dynamics impact the solar chromosphere and corona.

The simulation is initialized with a purely radial, bipolar magnetic field such that a transition from high to low plasma- occurs near the photospheric boundary. At present, the simulated coronal layer is passively heated to a fixed temperature equal to 10 times that of the photosphere. The simulation also includes a shallow, Newtonian-cooled chromospheric layer situated between the convection zone and corona.

Two dimensional simulations have been run far enough to assess details of the dynamics. Here a case with a Rayleigh number in the convection zone of $R=3 \times 10^{5}$, as displayed in Figure 1. The convective flows have structured the magnetic field to form flux sheets surrounded by inflowing fluid. These flows generate large currents in the high-b plasma there and can both eject flux into the low-b corona and retract it back.

In the corona, regions of reconnection near the outer boundary leads to strong current systems there as well as magnetoacoustic waves. These coronal wave possess a substantial, non-radial velocity field and are clearly modified by the structuring of the corona driven by the convective motions.

Self-consistently coupling a low- environment (such as our model corona) to the layer of convection allows the investigation of topics such as: how emergent bipoles affect the topology of the corona, how currents might be generated in coronal loop structures, how and where stresses build up that might lead to eruptive events, how helicity changes over time, etc. These models permit a detailed study of the energy budget and exchange between the photosphere, chromosphere and corona, which is essential for understanding the sources of coronal heating. We are currently exploring the parameter space of our model in two-dimensions to explore the range of possible behavior, and soon will begin serious exploration of the full, three-dimensional system.

\section{References}

DeRosa M. \& Hurlburt N. 2003 3D Stellar Evolution, ASP Conference Proceedings 99, eds. Sylvain Turcotte, Stefan C. Keller and Robert M. Cavallo, 293,229. 